\title{
Urachal Remnants and Anomalies in Children: Rationales of Surgery
}

\author{
(1) Beytullah Yağız \\ Ondokuz Mayıs University Faculty of Medicine, Department of Pediatric Surgery, Division of Pediatric Urology, Samsun, Turkiye
}

\section{What's known on the subject? and What does the study add?}

The prevelence of urachal anomalies are inconsistent in the literature due to employed imaging modality and inconsistent definitions. Major indications for surgery are presence of symptoms, infections and fear of future malignant transformation. After retrospectively evaluating our experience, we concluded that fistulography should not be performed routinely as it is hard to perform in children and does not add much to the management process. Voiding cystogram is recommended only in patients with suspected vesicoureteral reflux or if the results of the study may potentially alter the management process. Although potential future malignant transformation is still undetermined, malignancy should be considered as we found a neuroblastoma in the periurachal area.

\section{Abstract}

Objective: Urachal remnants are rare conditions in children with controversial management. Many of them present with complaints; however, the causality between the symptom and the condition is unclear, and a significant proportion of patients are incidentally identified. Meanwhile, indications of the surgical correction are controversial and the best way of surgical approach depends on the attending physician's experience. This study aimed to evaluate the results of surgically corrected urachal conditions in children.

Materials and Methods: This study included patients ( $n=21)$ who underwent surgery for urachal conditions between 2010 and 2020 . Age, presenting symptoms, radiological evaluation, surgical approach, and results, and histological results are retrospectively evaluated.

Results: Of the patients, 12 (57\%) were boys, and abdominal pain was the most common presenting symptom (43\%). The exact anomaly could not be classified in 8 patients, but classified as urachal sinus $(n=5)$, urachal cyst $(n=4)$, patent urachus $(n=2)$, urachal diverticulum ( $n=1)$, and neuroblastoma $(n=1)$ in the rest. Patients with umbilical discharge presented significantly younger than patients with abdominal pain or incidentally recognized patients $(2.4 \pm 1.2,7.2 \pm 1.17$, and $10.9 \pm 3.1$ years, respectively; $p<0.05)$. No operative complication is encountered. Histological examination revealed only 1 malignancy (neuroblastoma, 5\%).

Conclusion: Urachal anomalies in children are confusing conditions as they are rare, with unclear significance. Major indications of surgery are complications (infection, persisting drainage, or pain) and potential risk of malignant transformation. Surgery can be offered if complicated and the risk of malignancy appears to be low although not zero.

Keywords: Urachus, surgery, children

\section{Introduction}

Urachus is the fibrous remnant of the allantois, which is expected to become obliterated around birth. This process widely varies among individuals and may remain as a bulge at the apex of the bladder or may exhibit as a completely open tract between the bladder and umbilicus. Urachal anomalies are significantly rare and commonly classified as urachal cyst, patent urachus, urachal sinus, and urachal diverticulum (1).
However, as it is hard to define and classify a finding related to urachus in daily practice (radiological or clinical), Naiditch et al. (2) did not use the term "anomaly" and used "remnant" instead and mentioned the $5^{\text {th }}$ entity as "urachal remnant/chords". Urachal conditions usually present with complications or maybe incidentally identified during the investigation of relevant or irrelevant conditions. The exact incidence is hard to estimate as the definition of the physiological and pathological conditions differ among the studies, and most series lack surgical and

Correspondence: Beytullah Yağız MD, Ondokuz Mayıs University Faculty of Medicine, Department of Pediatric Surgery, Division of Pediatric Urology,

Samsun, Turkiye

Phone: +90 5336587502 E-mail: beytullahyagiz@gmail.com ORCID-ID: orcid.org/0000-0003-0882-1789

Received: 03.04 .2021 Accepted: 31.07 .2021

Cite this article as: Yağız B. Urachal Remnants and Anomalies In Children: Rationales of Surgery. J Urol Surg, 2022;9(1):63-67.

oCopyright 2022 by the Association of Urological Surgery / Journal of Urological Surgery published by Galenos Publishing House. 
histological confirmation. Thus, management is controversial as some recommend surgical removal, while others advocate a more conservative approach even in complicated cases, due to insufficient definitions and significant rarity $(1,3-5)$. This study aimed to retrospectively evaluate the clinical features and surgical and histological results of children with urachal conditions.

\section{Materials and Methods}

This study includes patients who underwent surgery for a urachal condition between January 2010 and April 2020. Institutional board approval is obtained from the Ethical Board on Clinical Studies of Ondokuz Mayıs University (IRB registry number: 2020/502). The patient's age, presenting symptoms, surgical approach, diagnostic tools, and histological results are retrospectively evaluated. Patients who are admitted with abdominal pain, umbilical discharge, and urinary tract infection were all regarded as symptomatic and only the cases encountered during surgery of an irrelevant condition are regarded as "incidental," because relating a symptom with the urachal pathology is highly subjective and causes significant bias.

\section{Statistical Analysis}

Numerical variables were expressed as mean \pm standard deviation depending on the distribution of variables. Categorical variables were expressed as numbers and percentages. The normality test of numerical variables was calculated with the Shapiro-Wilk test. One-Way analysis of variance with the post hoc Tukey test was used for statistical analysis of patients' age among admitting symptoms. Data were analyzed using the Statistical Package for the Social Sciences ${ }^{\circ}$ Statistics 20 software (IBM ${ }^{\circledast}$ Corp, Armonk, NY, USA).

\section{Results}

During the study period, 21 children underwent surgery for urachal anomalies. Of the patients, 12 (57\%) were boys and 9 $(43 \%)$ were girls. The presenting symptoms include abdominal pain $(n=9,43 \%)$, umbilical discharge $(n=7,33 \%)$, incidental diagnosis during surgery $(n=3,14 \%)$, prenatal diagnosis $(n=1$, $5 \%)$ and recurrent urinary tract infection $(n=1,5 \%)$.
The mean age at presentation was $2.4 \pm 1.2,7.2 \pm 1.17$, and $10.9 \pm 3.1$ years (mean \pm standard error) for the patients admitted for umbilical discharge, abdominal pain, and incidental cases, respectively. The patients admitted with umbilical discharge were significantly younger than those admitted with pain and incidentally diagnosed $(p<0.05)$ (Table 1).

Ultrasonographic (US) imaging yielded positive findings, which suggest a urachal condition in 14 patients (67\%), whereas 3 patients underwent additional magnetic resonance imaging (MRI) and 2 patients underwent surgery as clinical findings suggested an anomaly despite the negative US. Three patients were incidentally diagnosed during surgery for irrelevant conditions (Meckel's diverticulum, acute appendicitis, and ureteroneocystostomy, respectively). Avoiding cystourethrography (VCUG) was obtained in 8 patients before surgery but did not provide any additional information regarding the urachal anomaly. Unilateral vesicoureteral reflux was identified in 2 patients ( 1 resolved with conservative follow-up and the other was the one who had incidental diagnosis during ureteroneocystostomy). Of the 7 patients who presented with umbilical discharge, 3 underwent an attempt of fistulography, but all failed to demonstrate a patent urachus or urachal sinus.

Nine patients (43\%) underwent laparoscopic exploration while 12 $(57 \%)$ underwent open surgery by a median $(n=6)$, periumbilical smiling face $(n=2)$, Rockey-Davis $(n=2)$, Pfannenstiel $(n=1)$, and right upper quadrant transverse $(n=1)$ incisions (Figure 1). Simultaneous diagnostic cystoscopy was performed in 3 patients but did not yield any additional information or relief during surgery. Of the patients, 5 (24\%) received a cycle of antibiotics before surgical correction for a relevant infection or abscess.

The predominant histological features were fibromuscular tissue ( $n=12$; with 9 demonstrating uroepithelium), chronic inflammation $(n=4)$, fibroadipose tissue $(n=2)$, neuroblastoma $(n=1)$, connective tissue with mucinous glands $(n=1)$, and with uroepithelium $(n=1)$. The only malignancy in our series was the 2-month-old girl with neuroblastoma who presented with a prenatal diagnosis of urachal anomaly (5\%). The extraperitoneal location of the tumor above the bladder was confirmed by MRI and on surgical exploration (Figure 2). As radiological and laboratory findings were suggestive of a malignancy, the procedure was converted to open after diagnostic laparoscopy

Table 1. Presenting symptoms of the patients. The conditions recognized during an irrelevant surgery are considered as "incidental"

\begin{tabular}{|l|l|l|l|l|l|}
\hline & $\mathbf{n}$ & Mean (years) & Std. Error & Minimum (years) & Maximum (years) \\
\hline Umbilical discharge ${ }^{\mathrm{a}}$ & 7 & 2.39214 & 1.191299 & 0.288 & 9.333 \\
\hline Abdominal pain $^{\text {b }}$ & 9 & 7.20144 & 1.171635 & 0.481 & 11.750 \\
\hline Incidental b $^{\text {Total }}$ & 3 & 10.86067 & 3.076675 & 5.083 & 15.583 \\
\hline TSignificant at 5\% significance level, a,b: For all variables with the same letter, the difference between the means is not statistically significant & 15.583 \\
\hline
\end{tabular}


confirmed the preperitoneal location of the mass. The mass was located above the bladder between the medial umbilical ligaments and a small portion of the bladder wall was included in the excision. Histological examination revealed poorly differentiated neuroblastoma that demonstrated expansile protrusion into the detrusor muscle of the bladder (Figure 2). The major concerns about malignancy in these patients were the solid appearance on MRI and absence of infection signs.

Including the flap of full-thickness bladder wall into the excised specimen was necessary for 4 patients to ensure a clear margin that necessitated prolonged bladder drainage by urethral catheterization. No other morbidity or complication is encountered in these patients related to bladder wall violation.

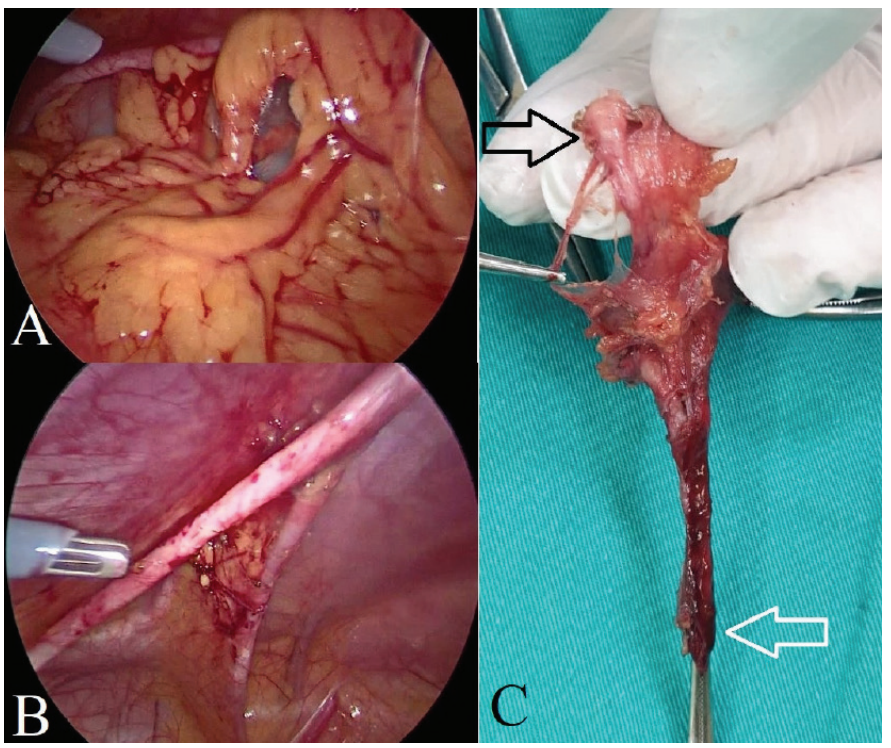

Figure 1. Laparoscopic view of a patent urachus with omental adhesions due to a previous infection (A). Major site of adhesion after omentum was released (B) and gross image of the specimen after excision (C). Black arrow indicates the caudal pole (bladder) and white arrow indicates the cranial pole (umbilical) of the tract (the tract is excised en bloc together with the median ligaments to ensure a clear margin)
Satisfactory classification of the condition was not possible in 8 patients (38\%) due to the retrospective design of the study and insufficient clear definitions of urachal anomalies. Five (38\%) patients were regarded as urachal sinus, $4(31 \%)$ as urachal cyst, $2(15 \%)$ as patent urachus, $1(8 \%)$ as urachal diverticulum, and $1(8 \%)$ as neuroblastoma (Figure 1).

No intraoperative or postoperative complication related to surgery was identified in any of the patients.

\section{Discussion}

Embryologically, urachus is a remnant tissue that is derived from allantois and usually obliterates after $18^{\text {th }}$ gestational weeks and remains as a fibrous cord or peritoneal fold (6). Urachal anomalies are rare conditions and their exact incidence is quite impossible to determine due to conflicting reports, unclear definitions, and poor understanding of the anomaly, but generally estimated as $1 \%(7,8)$. However, Ozbek et al. (9) found a space-occupying urachal lesion by US examination in 180 of 182 patients (99\%) who are admitted for irrelevant symptoms. Their prospective study revealed the mean diameter of the lesions as $13 \pm 5 \mathrm{~mm}$ ( $87 \%$ were ovoid; $n=156$ ) and sonographic protrusion into the bladder was demonstrated in 74\% of the patients $(n=133)$. Similarly, Cacciarelli et al. (10) identified an elliptical structure in 62 of 100 children by the US, but the significance of these images remains unidentified as only 1 patient underwent surgical excision and histological examination revealed a normal urachal remnant. Contrarily, Gleason et al. (7) revealed a 1.03\% incidence of urachal anomalies using various imaging modalities in their retrospective study, in which most of the conditions were incidentally diagnosed and only $8.3 \%$ underwent surgical excision.

The most specific symptom for a urachal anomaly is an umbilical lesion or discharge but a significant number of patients are recognized during evaluation for abdominal pain or urinary tract infection, and it is quite subjective to relate the presenting

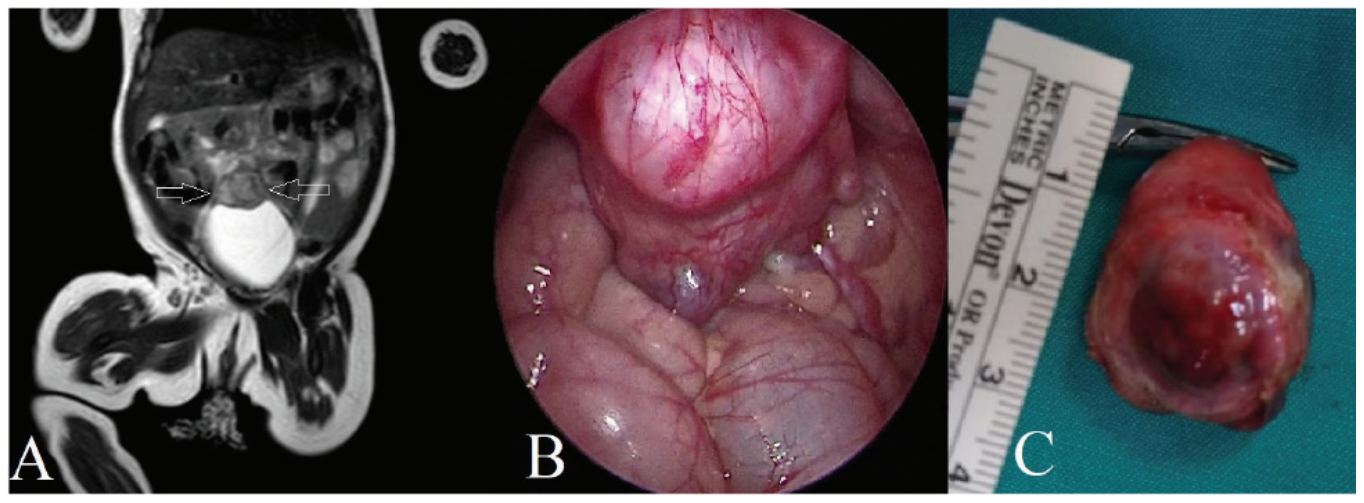

Figure 2. Coronal MRI section of the 2 months old patient with neuroblastoma. The heterogenous solid mass is indicated between the arrows in the apex of the bladder (A). Diagnostic laparoscopy revealed the preperitoneal mass between the median ligaments (B). Gross appearance of the excised specimen (C)

MRI: Magnetic resonance imaging 
symptoms with the urachal condition and coin the term "incidental" $(7,11)$. In our series, the most common presenting symptoms include abdominal pain, umbilical discharge, and incidental diagnosis, but we restricted the incidental term to only the patients who were recognized during surgery for other conditions. Relating the presenting symptom with the condition is quite difficult but significant as it may alter the decision on the management modality.

Most of the patients with urachal anomalies are incidentally recognized, and the US is the leading diagnostic tool. Urachal remnants, cysts, and diverticula can be seen as lesions that are ovoid or elliptical on US imaging (10). A sinus tract with or without air/fluid in it can also be demonstrated by the US but it's a subjective finding and reliability are unclear (12). Fistulography with a contrast medium may be performed when a patent urachus is suspected (i.e., in patients with umbilical discharge) but appears to be unreliable and demanding in our and others experience as canulation and visualization of a narrow lumen is challenging, and the cause of the umbilical discharge may be secondary to much more common lesions (i.e., umbilical granuloma) (12-14).

VCUG was also a commonly used imaging modality, especially for visualization of a urachal diverticulum or patent urachus $(1,7,15)$. However, VCUG did not provide additional information about the urachal condition or change the clinical management process in our 8 patients who underwent preoperative imaging. Therefore, we have abandoned VCUG for urachal anomaly diagnosis and recommend only for evaluation of other coexisting conditions, such as vesicoureteral reflux, like many others $(11,16)$.

Cross-sectional imaging modalities can be performed in selected patients when differential diagnosis, the extent, or nature of the anomaly are sought. We have performed MRI in 3 cases as all had solid mass images on the US, and histological examination of the surgical specimens revealed neuroblastoma that is located in the preperitoneal space above the bladder in one of our patients. A computerized tomography is a viable option; however, MRI appears as a superior imaging modality with improved soft-tissue resolution and the absence of radiation $(1,7)$.

Another controversial issue about the urachal anomalies is the management option (surgical or non-surgical). The decision of surgery is mainly driven by two concerns, complications and risk of malignancy. Complications of urachal anomalies, such as infection or discharge, are the least controversial issues as many recommend surgical excision when present $(1,7)$. However, some authors favor a more conservative approach to recommend surgery after multiple episodes of infection and advocate that complicated urachal anomalies can also be managed without surgery $(4,5,11)$. Opponents of nonsurgical management propose that urachal malignancy is rare in children and conservative management is safe $(2,7)$. Few cases of urachal malignancy are reported in children, with significantly diverse histological types from adults (as in our one case of neuroblastoma), which deepens the confusion on the origin of urachal tumors, and the risk of malignancy after childhood has insufficient data $(15,17,18)$. Identifying the origin of the malign tumors in children is quite impossible; however, the disparity in reported types suggests that they may arise from ectopic tissues located in the urachus, and ectopic neural crest cells would be responsible for the neuroblastoma in our 2-month-old patient.

As the prevalence of urachal malignancy is highest in the $6^{\text {th }}$ and $7^{\text {th }}$ decade, studies with long-term follow-up are lacking, which are necessary to identify the number of rare anomalies that transform into this rare urachal malignancy $(19,20)$. The length of follow-up is significantly short in most of the reports $(<5$ years) to safely conclude that risk of malignant transformation is low $(4,11)$. As significant surgical complications are rare, both in our series and in the literature, elective surgical excision appears reasonable in children to avoid a future aggressive malignancy, in which 5-year survival is $<50 \%(7,8,11,19)$. Conservative follow-up is a long road that requires patience, and most patients do not comply even with the annual followup controls (3).

The mode of surgical approach depends on the availability of instruments and familiarity with the techniques and can be performed by robotic, laparoscopic, or open approach. Laparoscopy became the technique of choice in recent years in our institution, but a paraumbilical smiling face incision provides sufficient exposure in selected patients ( $<2$ years) as the bladder is still an abdominal organ and its apex can easily reach the umbilicus when distended (Figure 3).

Therefore, we recommend elective surgery for symptomatic or complicated cases (infection, persisting umbilical discharge, and unexplained lower abdominal pain). However, in the absence of complications and symptoms, we inform the parents of the theoretically low but unclear possibility of malignancy and leave the final decision to them for patients without clinical or radiological findings suggesting malignancy.

\section{Conclusion}

Urachal anomalies remain an enigma for the surgeon, radiologist, and pathologist. Management is less controversial in symptomatic patients who present with umbilical discharge, infection signs, or mass lesions that are suspicious for malignancy. Major controversy persists for asymptomatic patients concerning the risk of malignancy as no long-term data is available regarding the actual risk of malignant transformation. 


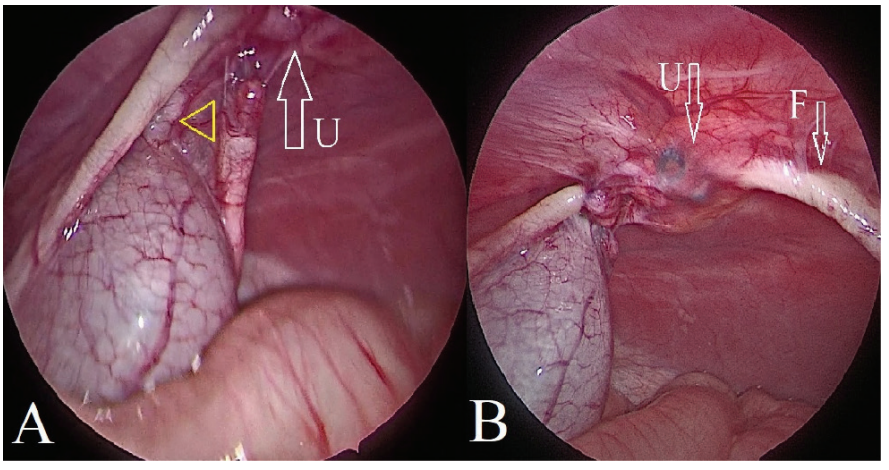

Figure 3. A patent urachus of a 5 months old infant was excised through a umbilical smiling face incision under laparoscopic vision. Note that dome of the bladder (yellow arrow head) reaches to the umbilicus $(U)$ when the bladder is distended. ( $U$; indicates umbilicus, $F$; indicates falciforme ligament and yellow arrow head indicates the urachus and the bladder dome)

\section{Ethics}

Ethics Committee Approval: Institutional board approval is obtained from the Ethical Board on Clinical Studies of Ondokuz Mayıs University (IRB registry number: 2020/502).

Informed Consent: Retrospective study.

Peer-review: Externally peer-reviewed.

Conflict of Interest: No conflict of interest was declared by the author.

Financial Disclosure: The author declare that they have no relevant financial.

\section{References}

1. Buddha S, Menias CO, Katabathina VS. Imaging of urachal anomalies. Abdom Radiol (NY) 2019;44:3978-3989.

2. Naiditch JA, Radhakrishnan J, Chin AC. Current diagnosis and management of urachal remnants. J Pediatr Surg 2013;48:2148-2152.

3. Ashley RA, Inman BA, Routh JC, Rohlinger AL, Husmann DA, Kramer SA. Urachal anomalies: a longitudinal study of urachal remnants in children and adults. J Urol 2007;178:1615-1618.

4. Lipskar AM, Glick RD, Rosen NG, Layliev J, Hong AR, Dolgin SE, Soffer SZ. Nonoperative management of symptomatic urachal anomalies. J Pediatr Surg 2010;45:1016-1019.
5. Ueno $T$, Hashimoto $H_{\text {, Yokoyama }}$, Ito $M$, Kouda $K$, Kanamaru $H$. Urachal anomalies: ultrasonography and management. J Pediatr Surg 2003;38:12031207.

6. Pazos HM, Costa WS, Sampaio FJ, Favorito LA. Structural and ontogenetic study of the urachus in human fetuses. Cells Tissues Organs 2010;191:422430.

7. Gleason JM, Bowlin PR, Bagli DJ, Lorenzo AJ, Hassouna T, Koyle MA, Farhat WA. A comprehensive review of pediatric urachal anomalies and predictive analysis for adult urachal adenocarcinoma. J Urol 2015;193:632-636.

8. Parada Villavicencio C, Adam SZ, Nikolaidis P, Yaghmai V, Miller FH. Imaging of the Urachus: Anomalies, Complications, and Mimics. Radiographics 2016;36:2049-2063.

9. Ozbek SS, Pourbagher MA, Pourbagher A. Urachal remnants in asymptomatic children: gray-scale and color Doppler sonographic findings. J Clin Ultrasound 2001;29:218-222.

10. Cacciarelli AA, Kass EJ, Yang SS. Urachal remnants: sonographic demonstration in children. Radiology 1990;174:473-475.

11. Dethlefs CR, Abdessalam SF, Raynor SC, Perry DA, Allbery SM, Lyden ER, Azarow KS, Cusick RA. Conservative management of urachal anomalies. $J$ Pediatr Surg 2019;54:1054-1058.

12. Boothroyd $A E$, Cudmore RE. Ultrasound of the discharging umbilicus. Pediatr Radiol 1996;26:362-364.

13. Nagasaki A, Handa N, Kawanami T. Diagnosis of urachal anomalies in infancy and childhood by contrast fistulography, ultrasound and CT. Pediatr Radiol 1991;21:321-323.

14. Zieger B, Sokol B, Rohrschneider WK, Darge K, Tröger J. Sonomorphology and involution of the normal urachus in asymptomatic newborns. Pediatr Radiol 1998;28:156-161.

15. D'Alessio A, Verdelli G, Bernardi M, DePascale S, Chiarenza SF, Giardina C, Cheli M, Rota G, Locatelli G. Endodermal sinus (yolk sac) tumor of the urachus. Eur J Pediatr Surg 1994;4:180-181.

16. Rich RH, Hardy BE, Filler RM. Surgery for anomalies of the urachus. J Pediatr Surg 1983;18:370-372.

17. Clapuyt P, Saint-Martin C, De Batselier P, Brichard B, Wese FX, Gosseye S. Urachal neuroblastoma: first case report. Pediatr Radiol 1999;29:320-321.

18. Gupta $S$, Bhaijee $F$, Harmon EP. Mucinous neoplasm arising in a urachal cyst: a first in the pediatric population. Urology 2014;83:455-456.

19. Bruins HM, Visser O, Ploeg M, Hulsbergen-van de Kaa CA, Kiemeney LA Witjes JA. The clinical epidemiology of urachal carcinoma: results of a large, population based study. J Urol 2012;188:1102-1107.

20. Mylonas KS, O Malley P, Ziogas IA, El-Kabab L, Nasioudis D. Malignant urachal neoplasms: A population-based study and systematic review of literature. Urol Oncol 2017;35:33.e11-33.e19. 